\title{
THE PLACE OF HYSTEROSCOPIC ENDOMETRIAL RESECTION FOR THE TREATMENT OF MENORRHAGIA
}

\author{
Mohammed Said, FRCOG; Ibrahim Hussein, FRCOG
}

The MISTLETOE (Minimally Invasive Surgical Techniques - Laser, EndoThermal Or Endoresection) study $^{(1)}$ of 10,000 patients provides a national database against which local practice can be compared. Mr Said, associate specialist in obstetrics and gynaecology, and $\mathrm{Mr}$ Hussein, consultant obstetrician and gynaecologist, who have been using this technique since 1992 at Furness General Hospital (FGH), compare their results with the standard.

\section{INTRODUCTION}

Heavy menstrual bleeding (HMB, also known as menorrhagia) is a significant cause of morbidity in premenopausal women in England and Wales. HMB is objectively defined as menstrual blood loss of more than $80 \mathrm{ml} /$ cycle, or menstrual bleeding lasting longer than seven days, over several consecutive cycles. However, in practice, the diagnosis is based on the woman's subjective assessment of blood loss.

It is estimated that 1 in 20 women in the United Kingdom (UK) aged 30-49 years consults her general practitioner (GP) each year with HMB - approximately 1.5 million women in England and Wales. Referrals for menstrual disorders account for about $20 \%$ of all referrals to specialist gynaecology services, placing a significant burden on secondary healthcare services. Medical treatment of this condition is either ineffective or poorly tolerated due to side effects, and for this reason hysterectomy has become the most frequently performed major operation in women of productive age. More than 47,000 hysterectomies were carried out in the NHS in England in 2000-2001. In at least half of those who undergo hysterectomies, menorrhagia is the main presenting problem. About half of all women who have a hysterectomy for menorrhagia have a normal uterus removed ${ }^{(2)}$.

The mortality rate associated with hysterectomy is low (0.4-1.1 per 1000 operations) when the indication is benign disease. Perhaps more worrying are the potential long term effects such as urinary and intestinal dysfunction, premature ovarian failure with consequent loss of protection against cardiovascular disease and possibly osteoporosis.

First generation endometrial ablation (EA) techniques were introduced almost 20 years ago as alternatives to hysterectomy. These techniques aim to reduce the menstrual bleeding by destroying (ablating) the entire thickness of the innermost layer of the uterus (the endometrium) and some of the underlying muscular layer (the myometrium) using electrical, thermal or laser energy. The most widely used first generation EA techniques are transcervical resection of endometrium (TCRE), using a loop diathermy electrode, and roller-ball ablation $(\mathrm{RB})$. The latter technique is used in FGH for treatment of menorrhagia.

\section{THE FURNESS EXPERIENCE OF TCRE}

We have been undertaking TCRE since 1992 and have experience of nearly 400 cases. Here we report our experience and an audit of our current practice using 100 sets of case notes compared with a national standard of some 10,000 cases (the MISTLETOE study $\left.{ }^{(1)}\right)$.

\section{Patient selection}

Women being referred for transcervical resection of the endometrium have to fulfil the following criteria before being judged suitable for this procedure:

- menstrual abnormalities that justify hysterectomy

- unresponsive to medical treatment

- intended family size must be complete

- uterine size must be smaller than equivalent of 12 week's pregnancy

- any submucous fibroid must be less than $5 \mathrm{~cm}$ in diameter

- the endometrium must be normal

- no concurrent gynaecological problems requiring hysterectomy (eg uterovaginal prolapse, endometriosis, pelvic inflammatory disease, cervical intraepithelial neoplasia)

Routine physical examination is supplemented by pelvic ultrasound scan and consent is obtained for laparoscopy or hysterectomy if indicated during the procedure. It is important also to have confirmation of benign endometrial histology. Four weeks before the procedure, preparation of the endometrium is commenced using a gonadotrophin hormone releasing agonist to ensure adequate depth of resection, minimise fluid absorption and improve the intraoperative view.

\section{Choice of anaesthesia}

Another advantage is that the technique can be carried out under local anaesthesia and sedation, though in our experience we have used general anaesthesia.

\section{Equipment and operative technique}

Endometrial resection was performed using standard hysteroscopic equipment, and a previously described technique $^{(3)}$. All operations were carried out by the same gynaecologist. Low viscosity ( $1.5 \%$ glycine) medium was used for uterine distention. The fluid balance was monitored continuously. A continuous flow resectoscope is generally used, fitted with a standard hysteroscope and cutting loop which cuts only to $3-4 \mathrm{~mm}$ (Figure 1) and a thin layer of myometrium should be removed to ensure there are no persistent glandular elements. 


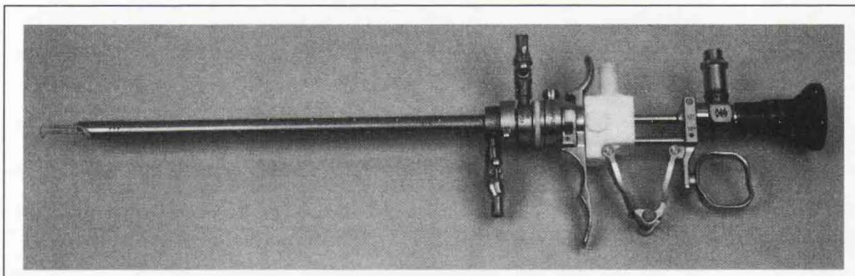

Figure I Continuous flow resectoscope

Usually, a cutting current of $70 \mathrm{~W}$ is used and the endometrium is systematically stripped around the cavity until the circular muscle fibres of the myometrium are seen. Usually, the endometrium of the fundus is resected first and then that around the tubal orifices, before stripping the remainder of the cavity. The strips of endometrium are sent for histological examination.

The average time of the procedure was less than 20 minutes and most of patients were fit for discharge in less than 24 hours.

\section{Follow-up}

The patients were routinely seen twice in six months and discharged if the results were satisfactory.

A sample of 100 endometrial resection case notes (between 1993-2000) were extracted for review to obtain the outcome after long term follow-up (five years) of TCRE procedure in $\mathrm{FGH}$ and to compare our practice with the national standards, such as the MISTLETOE study(1) in England and Wales.

\section{Results}

The majority of the patients $(56 \%)$ were in the age group 40 50 years (Figure 2 ). There were only two patients below 30 years of age.

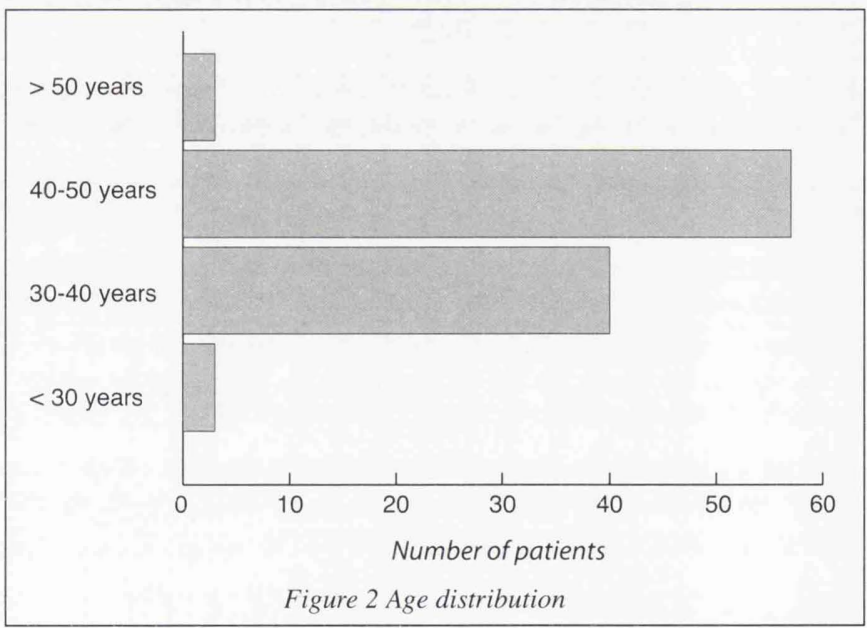

Nineteen per cent of the patients had previous medical treatment, but failed to respond. All the patients had hysteroscopic assessment prior to TCRE and it was noted that $2 \%$ of the patients had abnormal findings such as submucous fibroid or uterine polyp. Moreover, ultrasound scan was requested in $42 \%$ of the patients, in which $19 \%$ of those who had ultrasound scan showed abnormalities such as bulky or fibroid uterus.

As patients were warned that sterility cannot be guaranteed, simultaneous laparoscopic tubal sterilisation was carried out in 12 patients.

\section{Outcome}

After six months follow-up of the patients, only $11 \%$ reported lack of improvement in their periods; $65 \%$ of the patients noted lighter, shorter and less painful periods, with amenorrhoea being achieved in $24 \%$ of cases (Figure 3 ).

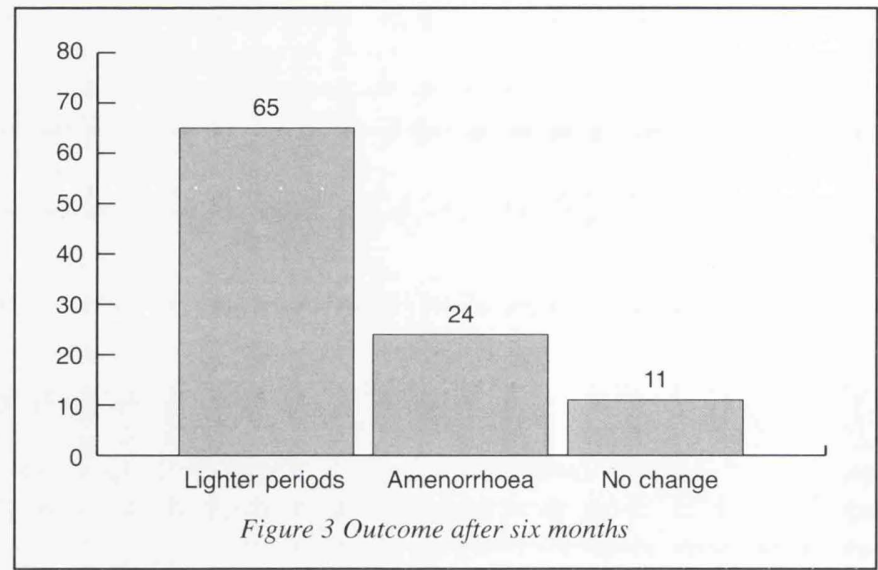

Long term outcome after five years showed that the success rate of surgery dropped to $73 \%$. While $2 \%$ of the patients opted for repeat TCRE, $25 \%$ of the patients ended by having hysterectomy (Figure 4).

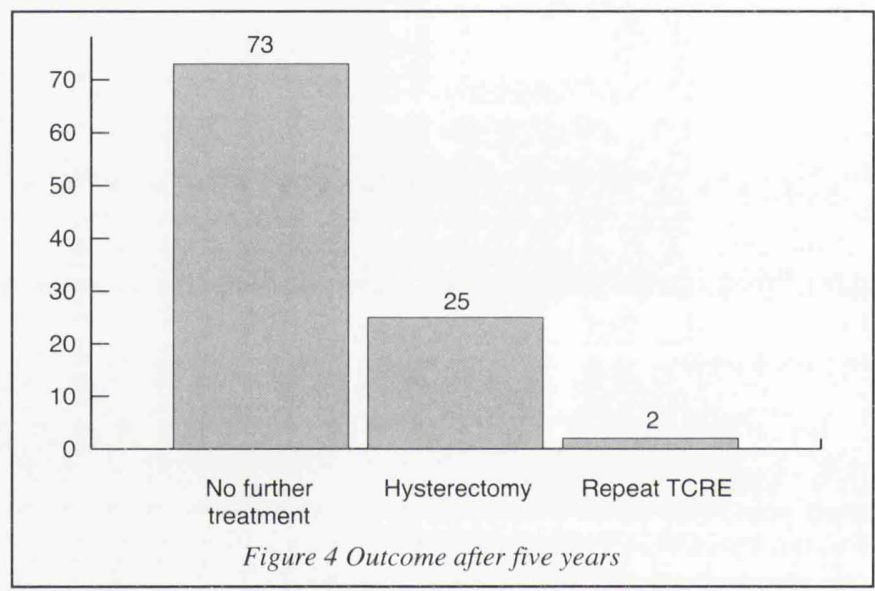

Two cases had repeat endometrial resection; one after eight months of the first surgery and the second after four years. The time interval of 25 cases which had hysterectomy after the first TCRE is shown in Figure 5.

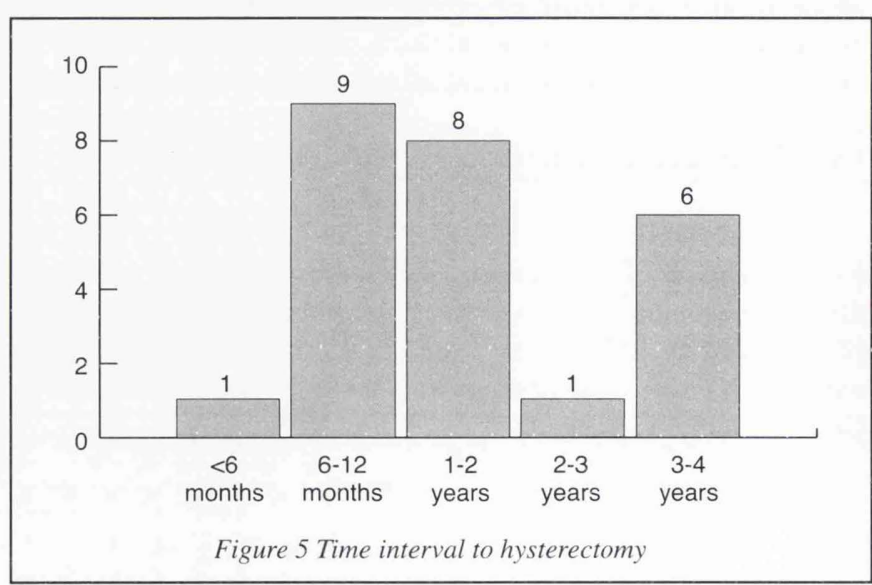

\section{Complications}

The overall complication rate was $5 \%$. The most serious one was one case of fluid overload (ie hypertension, hyponatraemia, neurological symptoms, haemolysis, and coma). The patient was one of the early cases operated upon since the introduction of TCRE. The patient had submucous fibroid, and the duration of the procedure was longer than 
average. She had a cardiac arrest at the end of the procedure and was resuscitated immediately by the anaesthetist and was managed in intensive care unit. Rapid correction of hyponatraemia was achieved by fluid restriction and diuresis. She made a good recovery without long term sequelae.

Minor complications such as postoperative haemorrhage, nausea, and infection are uncommon. No case of uterine perforation was recorded in the series.

Long term complications involving three cases of haematometra (a collection of blood and other menstrual fluid within the uterus) were diagnosed after four months, six months, and 12 months respectively. All three cases required dilatation and drainage under general anaesthesia.

\section{Patient satisfaction}

Questionnaires and pre-paid envelopes were distributed to a random sample of patients who had TCRE. The overall patient satisfaction for our unit was 76\% (Figure 6).

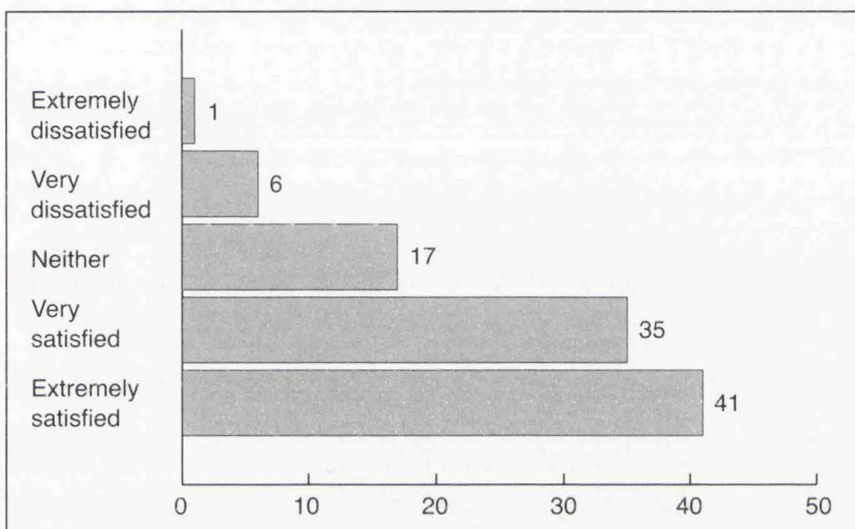

Figure 6 Patient satisfaction survey expressed as percentage

\section{DISCUSSION}

The results obtained of hysteroscopic endometrial resection in FGH are comparable to those previously reported by the MISTLETOE study ${ }^{(1)}$ in England and Wales (Table 1). There was no case of uterine perforation occurring during TCRE in FGH and none of the patients required emergency hysterectomy.

\begin{tabular}{|l|c|c|}
\hline & MISTLETOE & FGH \\
\hline Complications & $4.2 \%$ & $5 \%$ \\
\hline $\begin{array}{l}\text { Emergency hysterectomy } \\
\text { per } 1000\end{array}$ & 6 & 0 \\
\hline Failure Rate & $15.1 \%$ (1 year) & $\begin{array}{c}11 \%(6 \text { months) } \\
27 \%(5 \text { years })\end{array}$ \\
\hline Patient Satisfaction & $74 \%$ & $76 \%$ \\
\hline \multicolumn{2}{|c|}{ Table 1 Comparative audit of endometrial resection at FGH } \\
\hline
\end{tabular}

As with all endoscopic procedures, surgery is facilitated by video monitoring, with advantages in terms of operator comfort, magnification of operative field, maintenance of interest of the nurses and other staff, and not the least for teaching. The ability to confirm the completeness of surgery is a major advantage of hysteroscopic techniques, particularly endometrial resection.

After five years of follow-up, 27 patients (27\%) needed surgical intervention. The decreasing patient satisfaction over time may indicate that endometrial ablation is an especially good solution in older women who have a shorter period of time until menopause ${ }^{(4)}$. The re-intervention rates reported in the literature range from $3 \%$ to $34 \%$. However, comparison of these rates is hampered by differences in follow-up time and selection of patients ${ }^{(5)}$. In some studies, especially if small numbers are presented, it is not clear if the learning curve of the surgeons has been completed.

Hysterectomy in our study was performed because of menorraghia and metrorraghia. Probably the cause of failure was related to disorders of the uterus itself. Pathologists should be familiar with the tissue reactions to thermal ablation: proper recognition of the effects from the procedure will then be facilitated ${ }^{(4)}$.

Data on the cost of TCRE procedures were not collected in the present study. However, because the procedures were performed as day surgery and because the length of stay in hospital was short, the financial advantages of TCRE seem to be double fold compared with hysterectomy ${ }^{(6)}$.

The hazards of TCRE are potentially serious. In the present study, one patient (out of 384 TCRE cases carried out up to December 2004) had fluid overload syndrome. We use $1.5 \%$ glycine which is non-electrolytic, so that electrocoagulation current is not transmitted through them as would be the case with normal saline. Resection of endometrial tissue opens an extensive network of venous sinuses, which allows the irrigation fluid to be absorbed into the systemic circulation. Features may develop during the procedure or in the recovery room. This occurs in up to $3 \%$ of cases. It is similar to transurethral resection of the prostate (TURP) syndrome $^{(7)}$.

\section{The volume of fluid absorbed depends on:}

- prolonged duration of the procedure (associated with large uterus, or submucous fibroid)

- high pressure of the irrigation fluid bag above the patient (increased hydrostatic pressure driving the fluid intravenously)

- increased vascularity of the endometrium

An average of 10 to $30 \mathrm{ml}$ of fluid is absorbed per minute of resection time, amounting to up to $1800 \mathrm{ml}$ per hour. The glycine-containing irrigation solution is slightly hypoosmotic $(200 \mathrm{mosm} / \mathrm{l})$ and therefore the classical triad of features are:

1. Dilutional hyponatraemia. Encephalopathy and seizures may develop when the sodium concentration falls below $120 \mathrm{mmol} / 1$. Cerebral oedema may occur.

2. Fluid overload. This causes pulmonary oedema and cardiac failure.

3. Glycine toxicity. This inhibitory neurotransmitter causes depression of the level of consciousness and visual impairment at toxic levels.

If the patient is under general anaesthesia all of the symptoms and some of the signs are masked, and only unexplained tachycardia and hypotension may be present.

Achieving the correct balance for uterine distension is important. If the pressure is too low, the uterine cavity collapses and safe resection is not possible; if the pressure is too high, absorption of irregant fluid is enhanced possibly resulting in fluid overload. Strict guidelines concerning fluid 
balance are essential, which means in practice that fluid overload and all its sequelae are no longer a clinical problem, as surgery would be always terminated well before there was any danger to the patient ${ }^{(6)}$.

Second generation EA techniques have been introduced with the aim of providing simpler, quicker and more effective treatment options for HMB compared with first generation EA techniques and hysterectomy. These techniques are less operator dependent than the first generation techniques, but they rely heavily on the devices themselves to ensure safety and efficacy. The most frequently used second generation EA techniques in UK clinical practice are fluid-filled thermal balloon EA (TBEA) and microwave EA (MEA).

TBEA destroys the inner layers of the uterus by transferring heat from heated liquid within a balloon inserted into the uterine cavity. The devices involve an electronic controller, a single-use latex or silicone balloon catheter housing a heating element and two thermocouples, and an umbilical cable ${ }^{(2)}$. The balloon was inserted transcervically to touch the fundus and then was inflated with $5 \%$ dextrose in water (typically $10-15 \mathrm{ml}$ ) until the intrauterine pressure stabilised between $150-170 \mathrm{mmHg}$. The heater was then activated and it maintained the intra-balloon temperature at $87 \pm 5^{\circ} \mathrm{C}$ (Figure 7)

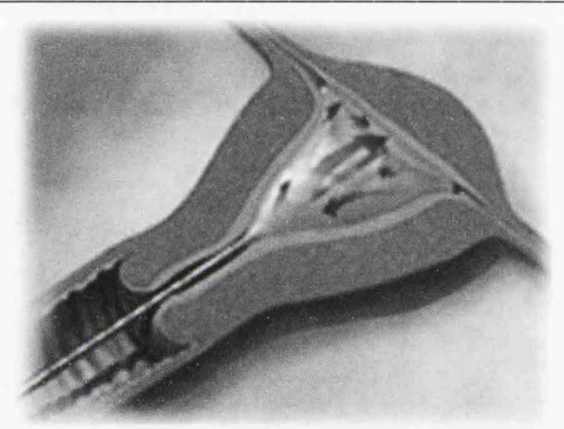

Figure 7 Fluid-filled thermal balloon EA (TBEA)

These techniques do not require direct visualisation of the uterine cavity, and can be carried out under either local or general anaesthesia.

TBEA cannot be used on women with large or irregular uterine cavities because the balloon must be in direct contact with the uterine wall to cause ablation ${ }^{(2)}$.

There is some concern about ablation techniques with respect to detection of endometrial cancer $^{(8)}$. Incidental case histories have been published of subsequent endometrial carcinomas that might be without the usual symptoms of abnormal vaginal bleeding because of cryptic location in the myometrium. Moreover, the endometrial cancer can be missed in the biopsy before ablation ${ }^{(9)}$. Therefore, endometrial resection was advocated in favour of the endometrial destruction, as it provides additional tissue for histological examination.

\section{CONCLUSION}

Endometrial resection is a valuable alternative procedure to hysterectomy in women with HMB (our audit demonstrated $89 \%$ and $73 \%$ success rates at six months and five years respectively).

The continued availability of first generation EA techniques is important because, for some women with HMB, these techniques may remain the most appropriate options (eg submucous fibroid).

The choice between different techniques should be made by the woman and the clinician responsible for her treatment, following informed discussion.

\section{ACKNOWLEDGEMENTS}

We acknowledge the invaluable contributions made by members of the Audit Department at FGH, especially to E Stewart.

\section{REFERENCES}

1. Overton C, Hargreaves J, Maresh M, A national survey of the complications of endometrial destruction for menstrual disorders: the MISTLETOE study. Contemporary review in Obstetrics \& Gynaecology 1998;9:209-16

2. National Institute for Clinical Excellence (NICE). Fluidfilled thermal balloon and microwave endometrial ablation techniques for heavy menstrual bleeding. April: 2004; pp.1-20

3. Salat-Baroux J, Hamou J, Antoine JM. La résection hystéroscopiquede l'endométre: une alternative à l'hystérectomie? Bull Acad Natl Med 1996;180:21393147

4. Bongers MY, Mol BW, Dukhuizen FP, Brolmann HA. Is Balloon Ablation as Effective as Endometrial Electroresection in the Treatment of Menorrhagia? J Laparoendosc Adv Surg Tech A 2000;10(2):85-92

5. Saridogan E, Bolaji II, Kadva A, Edmond A, Djahanbahch A, Djahanbahch 0. Long-term follow up of patients following transcervical endometrial resection. Gynaecol Endosc 1995;4:129-32

6. Broadbent M, Magos AL. Transcervical resection of endometrium. Contemp Rev Obstet Gynaecol 1992;4(1):21-8

7. Porter M, McCormick B. Anaesthesia for transurethral resection of the prostate (TURP). Update in Anaesthesia 2003;16(8):1-2

8. Copperman AB, DeChemey AH, Olive DL. A case of endometrial cancer following endometrial ablation for dysfunctional uterine bleeding. Obstet Gynecol 1993;82:640-2

9. Margolis MT, Thoen LD, Boike GM, Mercer LI, Keith LG. Asymptomatic endometrial carcinoma after endometrial ablation. Int J Gynaecol Obstet 1995;51(3):255-8 